\title{
The CD47-signal regulatory protein alpha (SIRPa) interaction is a therapeutic target for human solid tumors
}

\author{
Stephen B. Willingham ${ }^{\mathrm{a}, 1}$, Jens-Peter Volkmer ${ }^{\mathrm{a}, \mathrm{b}, 1}$, Andrew J. Gentlesc, Debashis Sahoo ${ }^{\mathrm{a}}$, Piero Dalerba ${ }^{\mathrm{a}, \mathrm{d}}$, \\ Siddhartha S. Mitra ${ }^{a}$, Jian Wang ${ }^{\text {e,f }}$, Humberto Contreras-Trujillo ${ }^{a}$, Robin Martin ${ }^{a}$, Justin D. Cohen ${ }^{a}$, Patricia Lovelace ${ }^{a}$, \\ Ferenc A. Scheeren ${ }^{a}$, Mark P. Chao ${ }^{a}$, Kipp Weiskopf ${ }^{a}$, Chad Tang ${ }^{a}$, Anne Kathrin Volkmer ${ }^{a}$, Tejaswitha J. Naik ${ }^{a}$, \\ Theresa A. Storm ${ }^{a}$, Adriane R. Mosley ${ }^{a}$, Badreddin Edris ${ }^{a}$, Seraina M. Schmid ${ }^{\text {g }}$, Chris K. Sun ${ }^{\mathrm{h}}$, Mei-Sze Chua ${ }^{\mathrm{h}}$, \\ Oihana Murillo ${ }^{a}$, Pradeep Rajendran ${ }^{a}$, Adriel C. Cha ${ }^{a}$, Robert K. Chin ${ }^{a}, \mathrm{i}$, Dongkyoon Kim ${ }^{\mathrm{a}}$, Maddalena Adorno ${ }^{\mathrm{a}}$, \\ Tal Raveh ${ }^{\mathrm{a}}$, Diane Tseng ${ }^{\mathrm{a}}$, Siddhartha Jaiswal ${ }^{\mathrm{a}}$, Per Øyvind Enger ${ }^{\mathrm{e}, \mathrm{f}}$, Gary K. Steinberg ${ }^{j}$, Gordon Lij, Samuel K. So ${ }^{\mathrm{h}}$, \\ Ravindra Majeti, ${ }^{a, k}$, Griffith R. Harsh ${ }^{\mathrm{i}, \mathrm{l}}$, Matt van de Rijn ${ }^{\mathrm{m}}$, Nelson N. H. Teng ${ }^{\mathrm{n}}$, John B. Sunwoo ${ }^{\mathrm{a}, \mathrm{l}}$, Ash A. Alizadeh ${ }^{\mathrm{a}, \mathrm{k}}$, \\ Michael F. Clarke ${ }^{\mathrm{a}, \mathrm{d}}$, and Irving L. Weissman ${ }^{\mathrm{a}, \mathrm{m}, 2}$
}

\begin{abstract}
${ }^{a}$ Institute for Stem Cell Biology and Regenerative Medicine and the Ludwig Cancer Center, ${ }^{b}$ Department of Urology, 'Stanford Research Initiative for Systems Biology of Cancer, ${ }^{d}$ Department of Internal Medicine, Division of Oncology, ${ }^{\mathrm{h}}$ Asian Liver Center, ${ }^{\mathrm{i}}$ Department of Radiation Oncology, ${ }^{\mathrm{j}}$ Department of Neurosurgery, ${ }^{k}$ Department of Internal Medicine, Division of Hematology, 'Department of Otolaryngology, Head and Neck Surgery, ${ }^{\mathrm{m}}$ Department of Pathology, and nepartment of Obstetrics and Gynecology, Stanford University Medical Center, Stanford, CA 94305; ${ }^{\mathrm{n}}$ Department of Neurosurgery, Haukeland University Hospital 5021 Bergen, Norway; ${ }^{f}$ Department of Biomedicine and Department of Neurosurgery, University of Bergen, 5009 Bergen, Norway; and ${ }^{9}$ Department of Obstetrics and Gynecology, University Women's Hospital Basel, 4031 Basel, Switzerland
\end{abstract}

Contributed by Irving L. Weissman, January 22, 2012 (sent for review December 13, 2011)

CD47, a "don't eat me" signal for phagocytic cells, is expressed on the surface of all human solid tumor cells. Analysis of patient tumor and matched adjacent normal (nontumor) tissue revealed that CD47 is overexpressed on cancer cells. CD47 mRNA expression levels correlated with a decreased probability of survival for multiple types of cancer. CD47 is a ligand for SIRPa, a protein expressed on macrophages and dendritic cells. In vitro, blockade of CD47 signaling using targeted monoclonal antibodies enabled macrophage phagocytosis of tumor cells that were otherwise protected. Administration of anti-CD47 antibodies inhibited tumor growth in orthotopic immunodeficient mouse xenotransplantation models established with patient tumor cells and increased the survival of the mice over time. Anti-CD47 antibody therapy initiated on larger tumors inhibited tumor growth and prevented or treated metastasis, but initiation of the therapy on smaller tumors was potentially curative. The safety and efficacy of targeting CD47 was further tested and validated in immune competent hosts using an orthotopic mouse breast cancer model. These results suggest all human solid tumor cells require CD47 expression to suppress phagocytic innate immune surveillance and elimination. These data, taken together with similar findings with other human neoplasms, show that CD47 is a commonly expressed molecule on all cancers, its function to block phagocytosis is known, and blockade of its function leads to tumor cell phagocytosis and elimination. CD47 is therefore a validated target for cancer therapies.

A voiding phagocytosis by tumor-associated macrophages is required for the growth and metastasis of solid tumors (1). Accumulating evidence suggests that cell-surface expression of CD47 is a common mechanism by which cells protect themselves from phagocytosis (1). CD47 expression is required to protect transfused red blood cells, platelets, and lymphocytes from rapid elimination by splenic macrophages (2-4). Mobilized hematopoietic stem cells protect themselves from phagocytosis by increasing CD47 expression as they pass through phagocyte-lined sinusoids and decrease it after relocating to marrow niches (5). Moreover, CD47 expression levels predicted the probability that hematopoietic stem cells would be phagocytosed while circulating (5).

CD47 is a widely expressed transmembrane protein with numerous functions (6). CD47 functions as a ligand for signal regulatory protein- $\alpha(\operatorname{SIRP} \alpha)$, a protein expressed on macrophages and dendritic cells (7). Upon binding CD47, SIRP $\alpha$ initiates a signaling cascade that results in the inhibition of phagocytosis (6). This "don't eat me" signal is transmitted by phosphorylation of the immunoreceptor tyrosine-based inhibition motifs present on the cytoplasmic tail of SIRP $\alpha$ (8). Subsequent binding and activation of SHP-1 and SHP-2 [src homology-2 (SH2)-domain containing protein tyrosine phosphatases] blocks phagocytosis, potentially by preventing the accumulation of myosin-IIA at the phagocytic synapse (9-12).

Here we show that CD47 is expressed on all human patient cancer cells tested. To our knowledge, CD47 is a unique nonhousekeeping cell-surface marker expressed by all human cancers. Increased CD47 mRNA expression levels in some solid tumors correlated with a decreased probability of patient survival. Monoclonal antibodies targeted to CD47 enabled the phagocytosis of patient solid tumor cells in vitro, inhibited the growth of orthotopically xenotransplanted human patient tumors, and prevented the metastasis of human patient tumor cells. These results establish CD47 as a critical regulator of innate immune surveillance.

\section{Results}

CD47 Is Expressed on Solid Tumor Cells. We evaluated CD47 expression on dissociated patient ovarian, breast, colon, bladder, glioblastoma, hepatocellular carcinoma, and prostate tumor cells by flow cytometry. Viability dyes and antibodies targeted to CD45, $\mathrm{CD} 31$, and $\mathrm{H}-2 \mathrm{~K}^{\mathrm{b} / \mathrm{d}}$ were used to exclude dead, nontumor (lineage), and mouse cells. CD47 expression was detected on nearly all

Author contributions: S.B.W., J.-P.V., A.J.G., D.S., P.D., S.S.M., J.W., H.C.-T., R. Martin J.D.C., P.L., M.P.C., K.W., C.T., A.K.V., T.A.S., B.E., S.M.S., P.R., M.A., T.R., D.T., S.J., P.O.E G.K.S., R. Majeti, M.v.d.R., J.B.S., A.A.A., M.F.C., and I.L.W. designed research; S.B.W., J.-P.V. A.J.G., D.S., P.D., S.S.M., J.W., H.C.-T., R. Martin, J.D.C., P.L., M.P.C., K.W., C.T., A.K.V., T.J.N., T.A.S., B.E., S.M.S., O.M., P.R., R.K.C., D.T., M.v.d.R., and A.A.A. performed research; S.B.W. J.-P.V., A.J.G., D.S., P.D., S.S.M., J.W., H.C.-T., R. Martin, J.D.C., P.L., F.A.S., K.W., A.K.V. T.J.N., T.A.S., A.R.M., B.E., S.M.S., C.K.S., M.-S.C., O.M., P.R., A.C.C., R.K.C., M.A., T.R., D.T., G.K.S., G.L., S.K.S., R. Majeti, G.R.H., M.v.d.R., N.N.H.T., J.B.S., A.A.A., and M.F.C. contrib uted new reagents/analytic tools; S.B.W., J.-P.V., A.J.G., D.S., S.S.M., J.W., H.C.-T., R. Martin, J.D.C., P.L., M.P.C., K.W., C.T., A.K.V., T.A.S., B.E., S.M.S., D.K., S.J., P.O.E., R. Majeti, M.v.d.R., N.N.H.T., J.B.S., A.A.A., M.F.C., and I.L.W. analyzed data; and S.B.W., J.-P.V., A.A.A., and I.L.W. wrote the paper.

Conflict of interest statement: S.J., M.P.C., R. Majeti, and I.L.W. filed U.S. Patent Application Serial No. 12/321,215 entitled "Methods for Manipulating Phagocytosis Mediated by CD47." I.L.W. owns Amgen Inc. stock and is a Director of Stem Cells, Inc.

Freely available online through the PNAS open access option.

${ }^{1}$ S.B.W. and J.-P.V. contributed equally to this work.

${ }^{2}$ To whom correspondence should be addressed. E-mail: irv@stanford.edu.

This article contains supporting information online at www.pnas.org/lookup/suppl/doi:10 1073/pnas.1121623109/-/DCSupplemental. 
A

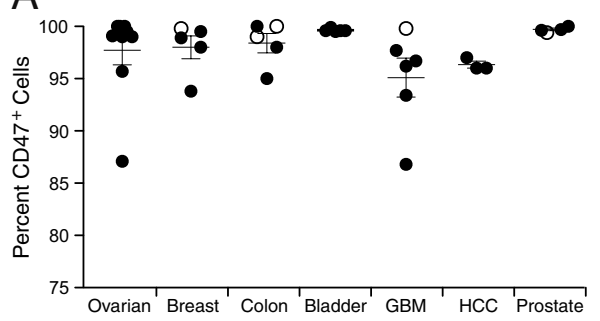

B

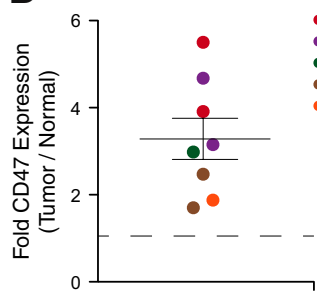

Fig. 1. CD47 is highly expressed on patient solid tumors. (A) Percentage of patient tumor (O) or normal $(O)$ cells that express CD47, as evaluated by flow cytometry, relative to an isotype-matched control. Each symbol represents a different tissue sample. (B) Tumor cells express more CD47 than matched adjacent normal cells, as evaluated by quantitative flow cytometry. Levels of CD47 expression were quantified on live, $\mathrm{CD}^{-} 5^{-}, \mathrm{CD}^{-} 1^{-}$, and $\mathrm{ESA}^{+}$cells. Analysis of the kidney samples was performed on live $\mathrm{CD} 45^{-}, \mathrm{CD} 31^{-}$cells. See also Fig. S1. GBM, glioblastoma multiforme; HCC, hepatocellular carcinoma. cancer cells from every primary and xenograft patient tumor sample evaluated (Fig. $1 A$; for representative analyses, see Fig. $\mathrm{S} 1 A)$. CD47 was also highly expressed on tumor samples when analyzed by immunofluorescence (Fig. S1B). As previously reported, CD47 was also detected on dissociated normal (noncancer) cells (Fig. 1A) (13-15). To evaluate the relative expression level of CD47 by tumor cells, we performed quantitative flow cytometry on dissociated cells from separate tumor or matched adjacent normal (nontumor) tissue specimens diagnosed by pathologists at Stanford University. On average, tumor cells expressed $\sim 3$.3-fold more CD47 than corresponding normal cells (Fig. 1B).

CD47 mRNA Expression Levels Predict Survival. We previously demonstrated that increased CD47 mRNA expression levels were correlated with poor clinical outcomes in patients with acute myeloid leukemia and non-Hodgkin's lymphoma $(16,17)$. To determine if CD47 mRNA expression levels were also a prognostic factor in human solid tumors, we analyzed gene-expression data from several previously described cohorts of patients with ovarian cancers, gliomas, and glioblastomas (Table S1) (18-25). In a univariate analysis, stratification of patients into "CD47 high" and "CD47 low" groups based on an optimum threshold revealed that high $C D 47$ mRNA expression levels were associated with a decreased probability of progression-free (Fig. $2 A$ and $B$ ) and overall (Fig. $2 C-F$ ) survival. These results suggest that CD47 expression levels may be a clinically relevant prognostic factor in some solid tumors.

Anti-CD47 Antibodies Enable Phagocytosis. We previously demonstrated that blockade of CD47-mediated SIRP $\alpha$ signaling using targeted monoclonal antibodies (mAbs) induced the phagocytosis of leukemia, lymphoma, and bladder cancer cells by human and mouse macrophages $(5,16,17,26)$. We expanded our evaluation of human solid tumors and tested the ability of purified blocking (clones B6H12.2 and Bric126) and nonblocking (clone 2D3) anti-CD47 mAbs to induce the phagocytosis of cells from dissociated patient ovarian, breast, and colon tumors, patientderived glioblastoma neurospheres, and colon cancer cell lines. In contrast to cells treated with isotype-matched mouse $\mathrm{IgG}$, anti-HLA, or nonblocking anti-CD47 (2D3) control mAbs, solid tumor cells treated with the blocking anti-human CD47 (hCD47) mAbs B6H12.2 and Bric126 were efficiently phagocytosed by human and mouse macrophages (Fig. $3 B$ and $C$; see Fig. $3 A$ for representative images and Movies S1 and S2 for videos demonstrating phagocytosis of human tumor cells following antihCD47 mAb treatment). To ensure anti-hCD47 therapy can also effectively target and eliminate cancer stem cells (CSCs), FACSpurified colorectal CSCs (lineage ${ }^{-} \mathrm{ESA}^{+} \mathrm{CD} 44^{+}$) were isolated from freshly dissociated xenograft tumors (27). Blocking antihCD47 mAbs induced the phagocytosis of purified colorectal CSCs by both human and mouse macrophages (Fig. 3D). These results suggest that $\mathrm{CD} 47$ is a legitimate therapy target, and that blocking anti-hCD47 mAbs may be effective therapeutic agents to inhibit solid tumor growth by enabling macrophages to eliminate both CSCs and their differentiated progeny.

Tumor-associated macrophages (TAMs) often aid tumor growth and may be functionally impaired and potentially ineffective at phagocytosis of tumor cells (28-30). To determine if
TAMs retain the ability to phagocytose tumor cells upon antihCD47 mAb treatment, we isolated TAMs $\left(\mathrm{F} 4 / 80^{+}\right)$and human tumor cells $\left(\mathrm{GFP}^{+}\right)$by FACS from single-cell suspensions prepared from large $\left(>1 \mathrm{~cm}^{3}\right)$ subcutaneous xenograft tumors established in NOD/SCID $/ \gamma$ (NSG) mice. These tumors were established with patient breast, bladder, or liver cancer cells previously transduced with a GFP encoding lentivirus. Purified tumor cells and TAMs were mixed in the presence of control IgG1 or anti-hCD47 mAbs and phagocytosis was evaluated by flow cytometry detection of GFP ${ }^{+}$TAMs. Blockade of CD47 increased TAM phagocytosis of human tumor cells (Fig. 3E; see Fig. S2 for representative analyses), demonstrating that TAMs can be converted into antitumor effectors by anti-hCD47 antibody therapy.

Anti-CD47 Antibodies Inhibit Tumor Growth. We hypothesized that anti-CD47 mAbs would enable the phagocytosis of xenotransplanted solid tumor cells, resulting in an inhibition or elimination of the developing tumor. Tumor cells from dissociated patient xenograft tumors (see Table S2 for tumor specimen details) were injected into immunodeficient NSG mice (see Table S3 for full experimental details). NSG mice lack B, T, and NK cells, but retain macrophages with phagocytic potential (Fig. 3). Unfortunately, the nonblocking 2D3 clone anti-hCD47 hybridoma is unavailable and

A

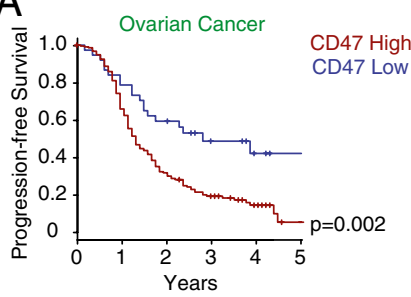

B

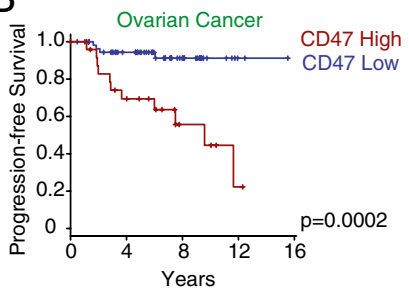

C
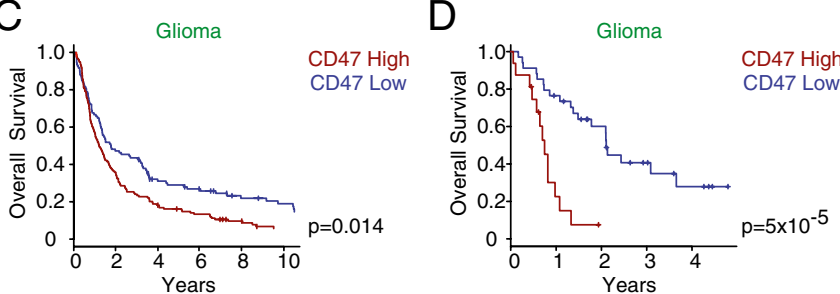

E
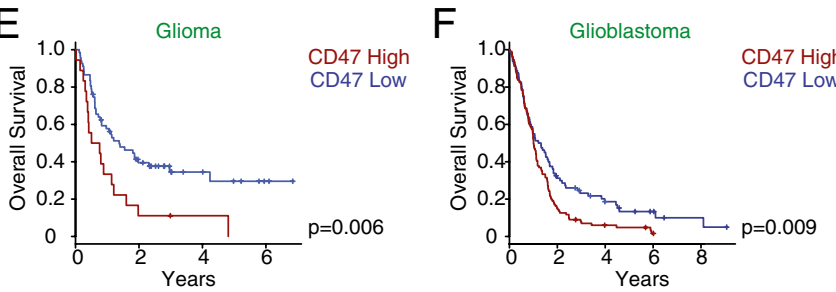

Fig. 2. CD47 mRNA expression levels may be a prognostic factor in solid tumors. Increased levels of CD47 mRNA expression were correlated with decreased probability of progression-free survival of ovarian cancer $(A$ and $B$ ) and overall survival of glioma $(C-E)$ and glioblastoma $(F)$. See also Table S1. 
A

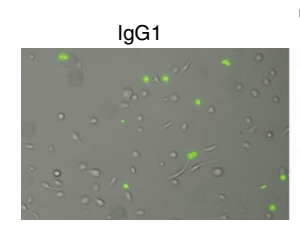

B

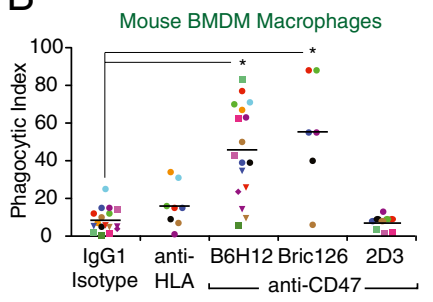

D

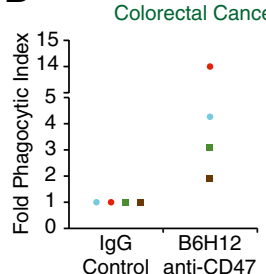

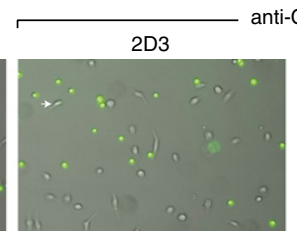

C

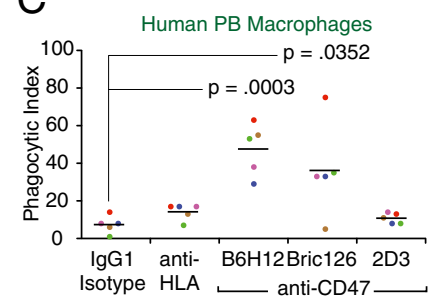

E
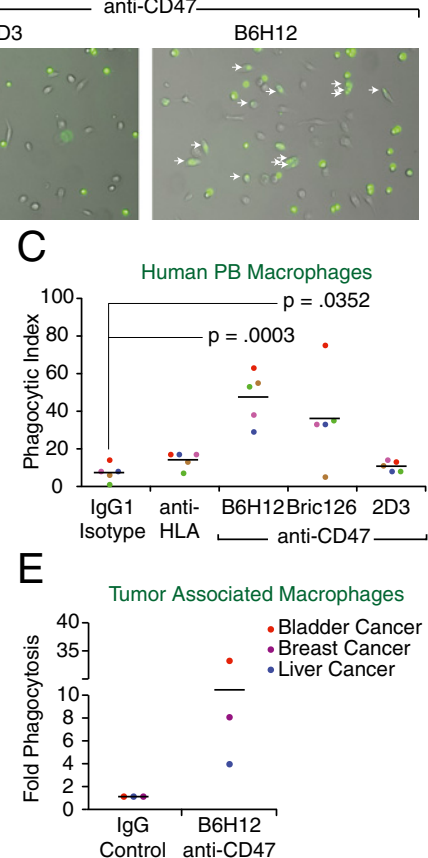

A

Control anti-CD47

$\mathrm{D}$

Fig. 3. Blockade of CD47-SIRP $\alpha$ interaction enables phagocytosis of patient tumor cells. $(A)$ Representative images of NSG bone marrow-derived macrophages (BMDM) phagocytosing human tumor cells following treatment with the indicated antibody. Arrows point to phagocytosed tumor cells. ( $B$ and $C$ ) Mouse BMDM $(B)$ and human peripheral blood- (PB) derived macrophages $(C)$ phagocytose patient tumor cells in the presence of blocking anti-CD47 mAbs (B6H12 and Bric126), but not control (IgG1 isotype and antiHLA) or nonblocking anti-CD47 antibodies (2D3). Each symbol color represents a different ovarian (circle), colon (square), glioblastoma (triangle), or breast (diamond) tumor sample. (D) Anti-CD47 mAbs enable the phagocytosis of colon CSCs (Lineage ${ }^{-} \mathrm{ESA}^{+} \mathrm{CD} 44^{+}$) by human PB derived (squares) and mouse BMDMs (circles). (E) Blocking anti-CD47 mAbs enable NSG tumor associated macrophages to phagocytose human tumor cells. Each symbol color represents a different tumor. Phagocytic Index indicates the number of tumor cells phagocytosed per 100 macrophages. ${ }^{\star} P<0.0001$. See also Fig. S2 and Movies S1 and S2. (Magnification, 100x.)

we were therefore unable include this antibody treatment as a control. Ovarian cancer xenotransplantation models were established in the peritoneal cavity (Fig. $4 A$ ). Prior transduction of these cells with a lentivirus designed to express GFP and Luciferase enabled the use of bioluminescent imaging to monitor tumor growth. Treatment with anti-hCD $47 \mathrm{mAbs}$ inhibited tumor growth as evidenced by bioluminescence imaging (Fig. $4 A$, see Fig. $4 B$ for representative images). Importantly, mice treated with antihCD47 mAbs demonstrated a dramatic increase in survival (Fig. $4 C$ ). Next, patient breast cancer cells were xenotransplanted into the mammary fat pad. After $8 \mathrm{wk}$, all (five of five) control IgGtreated mice developed large tumors but no tumors were palpable in the anti-hCD47 mAb-treated mice (Fig. 4D). Antibody treatments were discontinued and the mice were monitored for an additional 4 mo. No tumors developed in the mammary fat pad of anti-hCD47-treated mice, indicating that the mAb therapy had fully eliminated the breast cancer cells, including the CSCs (Fig. $4 D)$. Treatment of a second breast cancer sample resulted in a significant inhibition of tumor growth (Fig. $4 E$ ). No therapeutic benefit was observed following anti-CD47 mAb treatment of a third patient breast tumor (Fig. S3B). Patient colon cancer cells transduced with a GFP and luciferase-encoding lentivirus were injected subcutanteously onto the back of NSG mice. Several weeks after engraftment, anti-hCD47 antibody infusions resulted in a significant inhibition of tumor growth, as evaluated by bioluminescence (Fig. S3A) and tumor mass (Fig. $4 F$ ). Glioblastoma
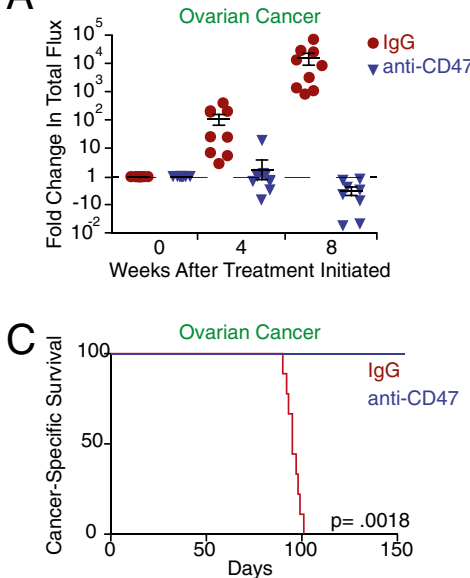

B
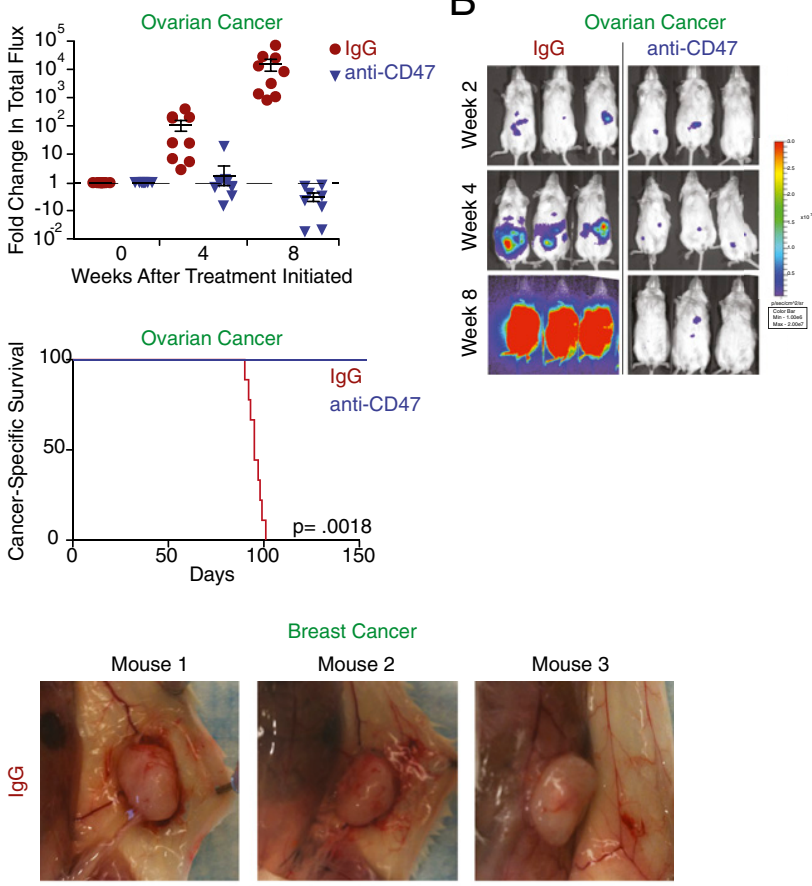

Breast Cancer

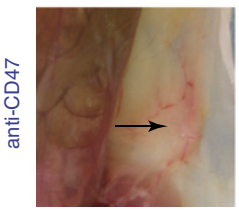

Mouse 2

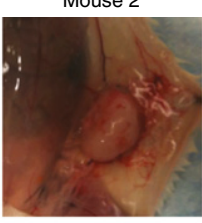

Mouse 3

E

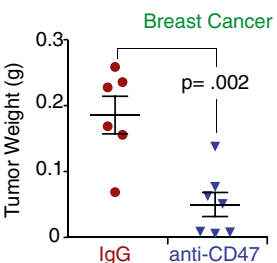

G
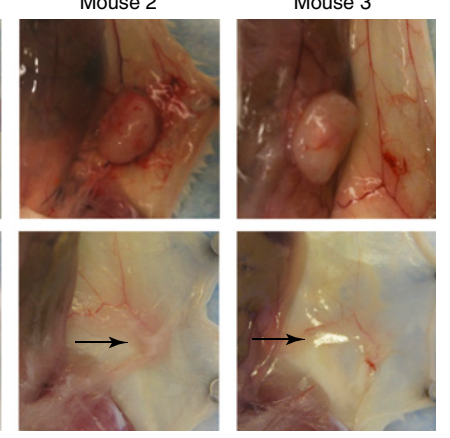

$\mathrm{F}$

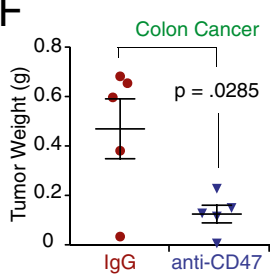

$\mathrm{H}$

Glioblastoma
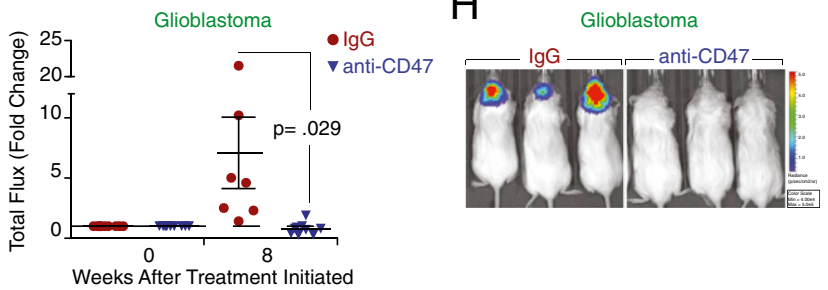

Fig. 4. Anti-CD47 mAbs inhibit tumor growth in xenotransplantation models. Anti-CD47 mAbs inhibit tumor growth as measured by bioluminescence activity $(A)$ and representative images $(B)$ and improve survival (C) of mice engrafted with ovarian cancer. $(D)$ Representative images of breast cancer engrafted mice treated with anti-CD47 mAbs. (E) Anti-CD47 mAbs inhibited the growth of a second breast cancer sample. $(F)$ Colon tumor growth was inhibited by anti-CD47 mAbs, as evaluated by tumor mass and bioluminescence (Fig. S3A). (G) Glioblastoma growth was inhibited by anti-CD47 mAb treatment. $(H)$ Representative bioluminescence images of treated mice. See also Fig. S3 and Tables S2 and S3.

cells transduced with the GFP Luciferase-encoding lentivirus were implanted into the left hemisphere of mouse brains. Subsequent intraperitoneal treatment with anti-hCD47 mAbs resulted in a significant reduction in tumor growth and possible elimination of the tumor in some mice (Fig. $4 G$ and $H$ ). In two additional xenotransplantation models of bladder and ovarian cancer, 
a substantial inhibition of tumor growth was observed in the majority of mice; anti-CD47 mAb treatment failed to halt tumor growth in rare cases (Fig. S3 $C$ and $D$ ). These results indicate that anti-CD47 antibodies can dramatically inhibit the growth of human solid tumors by blocking the ability of CD47 to transmit the "don't-eat-me" signal to macrophages.

Anti-CD47 Antibodies Prevent Tumor Metastasis. We hypothesized that inhibitory antibodies against CD47 would induce the phagocytosis of circulating tumor cells, or be therapeutically effective against newly seeded tumors, thereby preventing tumor metastasis. We identified a bladder cancer sample that spontaneously metastasized to the lymph nodes and lungs when engrafted subcutaneously onto the back of NSG mice. In contrast to previous experiments (Fig. 4), anti-hCD47 mAb therapy was not initiated until a large tumor mass was detected. Evaluated by gross examination, 24 lymph-node metastases were detected in control IgG-treated mice (Fig. $5 A$ and $B$ ). A single, substantially smaller, secondary lymph node was observed in one anti-hCD $47 \mathrm{mAb}$-treated mouse (Fig. $5 A$ and $B$ ). The presence of micrometastases in the lungs was also evaluated by microscopic analysis. Thirty-seven total micrometastases were detected in eight IgG-treated mice (Fig. $5 C$ and $D$ ). In contrast, solitary lung micrometastases were observed in three anti-hCD47-treated mice (Fig. $5 C$ and $D$ ). Anti-hCD47 mAb therapy also resulted in a significant inhibition of primary site tumor growth over the duration of the experiment (Fig. S4). The inhibitory effect of anti-CD47 mAbs on tumor metastasis was confirmed using a metastatic head and neck squamous cell carcinoma patient sample. When injected subcutaneously onto the back of NSG mice, this tumor rapidly metastasizes to mouse lymph
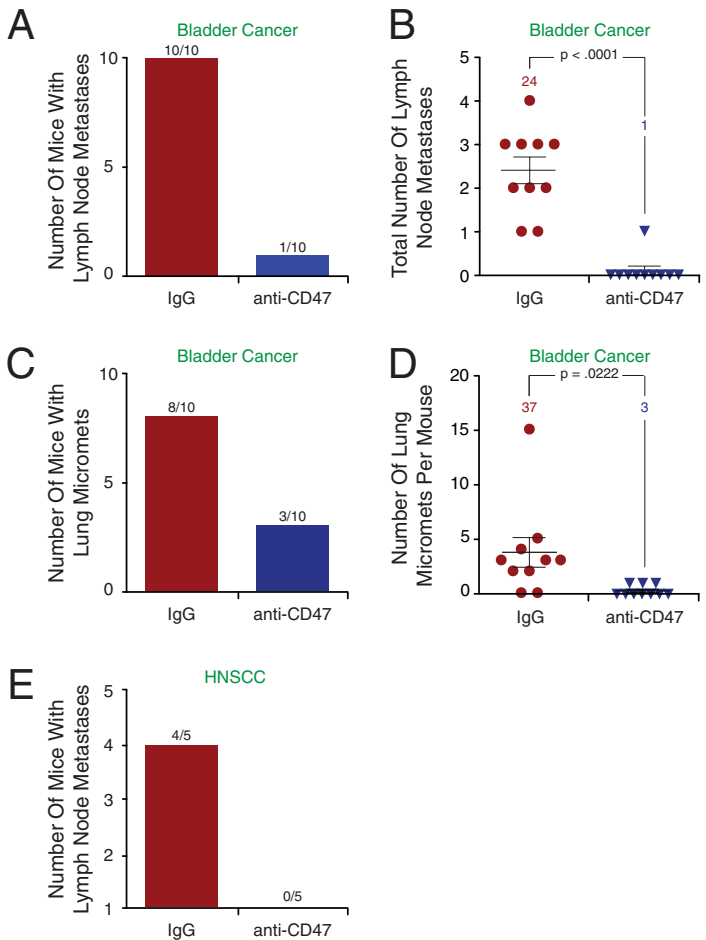

Fig. 5. Anti-CD47 mAbs prevent tumor metastasis. $(A)$ The number of mice exhibiting lymph node metastases in each cohort. $(B)$ The number of secondary lymph nodes detected in each mouse. The total number of secondary lymph nodes is indicated. (C) The total number of mice exhibiting lung micrometastases in each cohort. $(D)$ The number of micrometastases in the lungs of each mouse is shown. The total number of lung micrometastases is indicated. $(E)$ Anti-CD47 mAbs prevent head and neck squamous cell carcinoma cells from metastasizing to lymph nodes. The total number of secondary lymph nodes is shown. See also Fig. S4.

nodes. Because of the aggressive metastasis of this sample, antibody therapy was initiated before detection of a palpable primary tumor. Evaluated by gross examination, large secondary lymph nodes were observed in $80 \%$ (four of five) of the control $\mathrm{IgG}$ treated mice, but zero (zero of five) metastatic lesions were detected in mice following anti-hCD47 mAb therapy (Fig. 5E). These results indicate that CD47-targeted antibodies will not only inhibit primary tumor growth, but may also be effective in preventing tumor metastasis.

Anti-Mouse CD47 Antibodies Inhibit Tumor Growth in Immune Competent Hosts. To validate the safety and efficacy of targeting CD47 in fully immune competent hosts, we engrafted 50,000 MT1A2 mouse breast cancer cells into the mammary fat pad of syngeneic FVB mice (31). Upon detection of palpable tumors, $400 \mu \mathrm{g}$ of control IgG or anti-mouse CD47 (mCD47) mAbs were injected into the mammary fat pad every other day (Fig. $6 \mathrm{~A}$ ). This antibody dose was chosen to reveal any potential toxicity and is not necessarily indicative of the minimal effective dose. After $30 \mathrm{~d}$, tumors were resected and weighed. The anti-mCD47 mAb clone MIAP410 (mouse IgG1) significantly inhibited tumor growth (Fig. 6B) (32). A second anti-mCD47 antibody, clone MIAP301 (rat IgG2A) produced a modest, yet insignificant, inhibition of tumor growth (Fig. 6B) (33). Whereas extensive tumor burden with sparse lymphocytic infiltrate can be viewed in control IgG-treated tumors, Stanford University pathologists

A

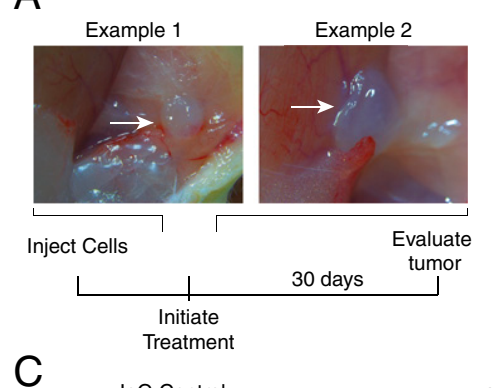

B

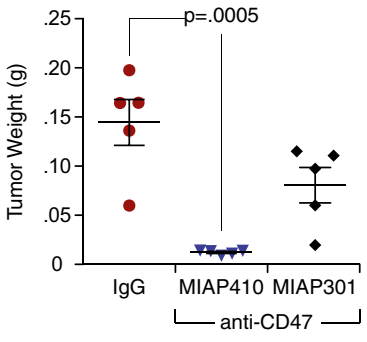

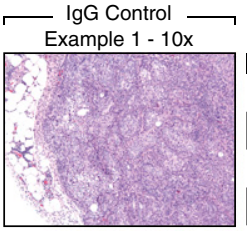

Example 2 - 60x

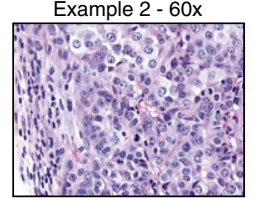

D

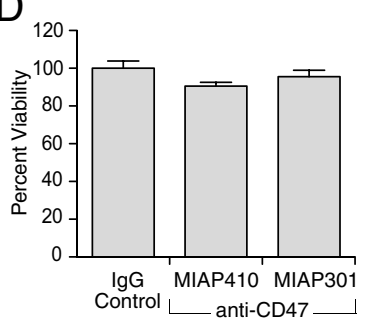

Fig. 6. Anti-CD47 antibodies inhibit tumor growth in immune competent hosts. (A) Representative images of MT1A2 breast tumors at initiation of anti-CD47 mAb therapy. (B) Anti-mouse CD47 mAbs inhibit MT1A2 tumor growth. (C) Representative H\&E images of IgG or anti-CD47 mAb treated tumors. $(D)$ Anti-CD47 mAbs $(10 \mu \mathrm{g} / \mathrm{mL})$ do not initiate cell death of MT1A2 cells. See also Fig. S5 and Table S4. (Magnification, 7.8×.) 
observed only isolated tumor cell nests surrounded by substantial inflammatory cell infiltrates anti-mCD47 treated tumors (Fig. $6 C)$. Importantly, the therapeutic anti-mCD47 mAbs produced no unacceptable toxicity over the course of the experiment, despite having spread systemically (Fig. S5). These experiments were analyzed at the end of the therapeutic regimen; acute infusion of these antibodies led to a short-term anemia (Fig. S6). A similar lack of toxicity was observed 1 wk after administering anti-mCD47 antibodies via intraperitoneal injection into tumor free BALB/c mice (Table S4). No increase in cell death was observed when MT1A2 cells were treated overnight with antiCD47 antibodies, suggesting that direct toxicity is unlikely to be the primary mechanism by which the anti-CD47 antibodies produce a therapeutic effect (Fig. 6D).

\section{Discussion}

Here we demonstrate that expression of CD47 is a general mechanism used by human patient solid tumor cells to evade phagocytosis. Blocking mAbs that disrupt the interaction between CD47 and SIRP $\alpha$ enabled the phagocytosis of solid tumor cells in vitro and inhibited tumor growth in several orthotopic xenotransplantation models. Moreover, anti-CD47 antibodies prevented the formation of tumor metastases. These results establish CD47 as a therapeutic target on solid tumor cells.

A concern in translating this therapy to human application is the potential for toxicity. CD47 is highly expressed on tumor cells, but also at varying levels on normal (nontumor) cells (6). However, here we demonstrate that blockade of CD47 in immune competent mice produces an effective antitumor response without unacceptable toxicity, albeit with a temporary anemia (Fig. 6, Fig. S6, and Table S4). In a previous report, administering therapeutic doses of blocking anti-mouse CD47 mAbs (clone MIAP301) to normal C57BL/6 mice produced no significant toxic effect except isolated neutropenia (17). In both studies, mice were treated with doses of anti-CD47 mAbs (200-400 $\mu \mathrm{g})$ that may be in far excess of the minimal effective dose. Furthermore, antiCD47 mAbs failed to induce phagocytosis of normal (noncancer) cells in vitro $(16,17)$. It is inferred from this finding that normal healthy tissues lack a secondary prophagocytic "eat me" signal and, in the absence of CD47-SIRP $\alpha$ signaling, are not subject to phagocytosis. Several prophagocytic signals have been identified, including cell surface calreticulin and phosphatidylserine $(34,35)$. Calreticulin interacts with LDL-receptor-related protein 1 on macrophages and is required for the phagocytosis of tumor cell lines following neutralization of the CD47-SIRP $\alpha$ interaction (36, 37). Cell surface calreticulin is present on a subset of cells in all human leukemias, lymphomas, and solid cancers, but not on nontumor cells (37). The presence of prophagocytic signals on tumor cells may provide a therapeutic window in which to administer anti-CD47 mAbs without causing phagocytosis of healthy cells that lack these "eat me" signals.

Thus far, we have evaluated the efficacy of anti-CD47 mAbs to inhibit solid tumor growth as a monotherapy. In our experiments, the efficacy of anti-CD47 therapy was inversely correlated with tumor size at the onset of treatment. Therefore, anti-CD47 antibody therapy may be most effective after the primary mass has been maximally debulked through cytoreductive surgery. Debulking can also be accomplished by chemotherapy or radiotherapy. However, caution must be used in applying antiCD47 therapy in the context of ongoing or recent cytotoxic or inflammatory therapy, as the translocation of calreticulin to the cell surface upon conditions of cell stress may render normal cells susceptible to phagocytosis by nearby macrophages $(38,39)$.

The therapeutic efficacy of anti-CD47 mAbs may be further enhanced when used concurrently with a second antitumor antibody. In particular, antibodies, such as trastuzumab and cetuximab, which induce antibody-dependent cell-mediated cytotoxicity through the Fc receptor on macrophages, may transmit an "eatme" signal that enhances the ability of anti-CD47 mAbs to induce phagocytosis (40). Such a strategy has already proven effective in xenotransplantation models of non-Hodgkin's lymphomas, where the combination of anti-CD47 and rituximab antibodies produced a synergistic induction of phagocytosis and therapeutic response (16).

Metastasis is the primary reason for failure of local therapies, such as surgery or radiotherapy (41). Anti-CD47 mAb therapy not only inhibited the growth of primary site tumors, but also prevented the formation of tumor metastases in the lymph nodes and lungs, or eliminated them as microtumors. Circulating tumor cells may even be particularly vulnerable to anti-CD47 mAbs if they rely on transmitting a "don't eat me" signal to perivascular macrophages for entry into blood vessels or tether to macrophages for assistance in migration $(1,42)$. It is also possible that the preventative effect on tumor metastasis is because of disrupting an interaction between CD47 and integrins. CD47 was initially identified as integrin-associated protein and has been shown to bind the $\alpha_{v} \beta_{3}, \alpha_{\text {IIb }} \beta_{3}, \alpha_{2} \beta_{1}$ integrins $(6,43)$. The B6H12.2 antibody may affect the ability of CD47 to interact with these integrins, thereby inhibiting their ability to adhere and migrate (44). Future studies will address the ability of anti-CD47 $\mathrm{mAbs}$ to eliminate established tumor metastases before and following surgical resection of the primary tumor, mimicking treatment of metastatic disease in the clinical setting.

Emerging evidence suggests TAMs support tumor progression and metastasis $(1,28-30,45)$. TAMs participate in the development of a microenvironment conducive to tumor growth through remodeling of extracellular matrix and release of factors that promote cell proliferation, angiogenesis, and migration (46). Here, we demonstrated that blockade of CD47 signaling enables TAMs to attack tumor cells that they would otherwise disregard (Fig. 3E). Given that TAMs are present in large numbers within tumors (46), it's possible that anti-CD47 antibody therapy has the potential to restore TAM immunosurveillance and fundamentally alter the role of macrophages in tumor biology.

In conclusion, we have found that CD47 is expressed on a wide range of human solid tumors, perhaps as the one constant change in all human aggressive neoplasms. We have also validated one function of CD47 on these cancers, as a "don't eat me" signal. CD47 therefore serves as an attractive target for cancer therapies. We have demonstrated that monoclonal antibodies that block CD47 are effective for treating human solid tumors in vitro and in vivo. We anticipate that these findings will extend to all modalities that interfere with the CD47-SIRPa interaction.

\section{Materials and Methods}

See SI Materials and Methods for detailed discussion. All animal procedures were approved by the Administrative Panel on Laboratory Animal Care at Stanford University.

Human Samples. Tissue specimens were obtained from consented patients as approved by Stanford University Institutional Review Board protocols. Colorectal cancer samples were obtained as previously described (27). Stanford University pathologists defined tumor and matched adjacent normal (nontumor) tissue specimens.

Survival Data Analysis. CD47 expression was dichotomized in large ( $n \geq 50$ samples) solid tumor datasets for which clinical annotations were available. The Maxstat package (version 0.7-13) (47) of the R programming language (version 2.11) was used to define high- and low-CD47 groups. Log-rank $P$ values and hazard ratios with $95 \%$ confidence intervals $(\mathrm{Cl})$ were derived by Kaplan-Meier analyses. For Affymetrix arrays, data were normalized from raw CEL files using MAS5 with a custom chip definition file-mapping array oligonucleotides to Entrez gene identifiers (48).

In Vitro Phagocytosis Assay. For in vitro phagocytosis assay, $5 \times 10^{4}$ macrophages were plated per well in a 24-well tissue-culture plate. Tumor cells were labeled with $2.5 \mu \mathrm{M}$ carboxyfluorescein succinimidyl ester (CFSE) according to the manufacturer's protocol (Invitrogen). Macrophages were incubated in serum-free medium for $2 \mathrm{~h}$ before adding $2 \times 10^{5}$ CFSE-labeled live tumor cells. The indicated antibodies $(10 \mu \mathrm{g} / \mathrm{mL})$ were added and incubated for $2 \mathrm{~h}$ at $37^{\circ}$. Macrophages were repeatedly washed and subsequently imaged with an inverted microscope (Leica DMI6000B). The phagocytic index was calculated as the number of phagocytosed $\mathrm{CFSE}^{+}$cells per 100 macrophages. For ex vivo assays, TAMs ( $\mathrm{F} 4 / 80^{+}$; eBioscience) and human tumor cells $\left(\mathrm{GFP}^{+}\right)$were isolated 
by FACS from single-cell suspensions prepared from large $\left(>1 \mathrm{~cm}^{3}\right)$ subcutaneous xenograft tumors established in NSG mice. Purified TAMs and tumor cells and were mixed in the presence of control lgG1 or anti-CD47 (B6H12) antibody $(20 \mu \mathrm{g} / \mathrm{mL})$ and incubated for $2-4 \mathrm{~h}$. Phagocytosis was then determined by flow cytometry detection of GFP' TAMs.

Antibody Preparation, Flow Cytometry Analysis, and Cell Sorting. The antihCD47 (B6H12) hybridoma was obtained from the ATCC. Hybridoma cells were cultured under standard conditions and antibodies were purified by Protein G. For quantification of CD47 expression, cells were labeled with a saturating concentration of a 1:1 phycoerythrin- (PE) conjugated anti-CD47 antibody (BD Pharmingen) and analyzed using a BD LSR Fortessa Analyzer. BD QuantiBRITE PE beads (BD Pharmingen) were analyzed the same settings and conditions as the patient samples. Median absolute CD47 antibody binding for each sample was determined from a calibration curve constructed

1. Jaiswal S, Chao MP, Majeti R, Weissman IL (2010) Macrophages as mediators of tumor immunosurveillance. Trends Immunol 31:212-219.

2. Oldenborg PA, Gresham HD, Lindberg FP (2001) CD47-signal regulatory protein alpha (SIRPalpha) regulates Fcgamma and complement receptor-mediated phagocytosis. J Exp Med 193:855-862.

3. Oldenborg PA (2004) Role of CD47 in erythroid cells and in autoimmunity. Leuk Lymphoma 45:1319-1327.

4. Blazar BR, et al. (2001) CD47 (integrin-associated protein) engagement of dendritic cell and macrophage counterreceptors is required to prevent the clearance of donor lymphohematopoietic cells. J Exp Med 194:541-549.

5. Jaiswal S, et al. (2009) CD47 is upregulated on circulating hematopoietic stem cells and leukemia cells to avoid phagocytosis. Cell 138:271-285.

6. Brown EJ, Frazier WA (2001) Integrin-associated protein (CD47) and its ligands. Trends Cell Biol 11:130-135.

7. Jiang $P$, Lagenaur CF, Narayanan $V$ (1999) Integrin-associated protein is a ligand for the P84 neural adhesion molecule. J Biol Chem 274:559-562.

8. Oldenborg PA, et al. (2000) Role of CD47 as a marker of self on red blood cells. Science 288:2051-2054.

9. Kharitonenkov A, et al. (1997) A family of proteins that inhibit signalling through tyrosine kinase receptors. Nature 386:181-186.

10. Fujioka Y, et al. (1996) A novel membrane glycoprotein, SHPS-1, that binds the SH2 domain-containing protein tyrosine phosphatase SHP-2 in response to mitogens and cell adhesion. Mol Cell Biol 16:6887-6899.

11. Timms JF, et al. (1998) Identification of major binding proteins and substrates for the SH2-containing protein tyrosine phosphatase SHP-1 in macrophages. Mol Cell Biol 18 3838-3850.

12. Tsai RK, Discher DE (2008) Inhibition of "self" engulfment through deactivation of myosin-II at the phagocytic synapse between human cells. J Cell Biol 180:989-1003.

13. Mateo V, et al. (2002) Mechanisms of CD47-induced caspase-independent cell death in normal and leukemic cells: link between phosphatidylserine exposure and cytoskeleton organization. Blood 100:2882-2890.

14. Rosales C, Gresham HD, Brown EJ (1992) Expression of the 50-kDa integrin-associated protein on myeloid cells and erythrocytes. J Immunol 149:2759-2764.

15. Mawby WJ, Holmes CH, Anstee DJ, Spring FA, Tanner MJ (1994) Isolation and characterization of CD47 glycoprotein: A multispanning membrane protein which is the same as integrin-associated protein (IAP) and the ovarian tumour marker OA3. Bio chem J 304:525-530.

16. Chao MP, et al. (2010) Anti-CD47 antibody synergizes with rituximab to promote phagocytosis and eradicate non-Hodgkin lymphoma. Cell 142:699-713.

17. Majeti R, et al. (2009) CD47 is an adverse prognostic factor and therapeutic antibody target on human acute myeloid leukemia stem cells. Cell 138:286-299.

18. Tothill RW, et al.; Australian Ovarian Cancer Study Group (2008) Novel molecular subtypes of serous and endometrioid ovarian cancer linked to clinical outcome. Clin Cancer Res 14:5198-5208.

19. Marchini S, et al. (2008) Analysis of gene expression in early-stage ovarian cancer. Clin Cancer Res 14:7850-7860.

20. Chung $\mathrm{CH}$, et al. (2004) Molecular classification of head and neck squamous cell carcinomas using patterns of gene expression. Cancer Cell 5:489-500.

21. Gravendeel LA, et al. (2009) Intrinsic gene expression profiles of gliomas are a bette predictor of survival than histology. Cancer Res 69:9065-9072.

22. Nutt $\mathrm{CL}$, et al. (2003) Gene expression-based classification of malignant gliomas correlates better with survival than histological classification. Cancer Res 63:1602-1607.

23. Freije WA, et al. (2004) Gene expression profiling of gliomas strongly predicts survival. Cancer Res 64:6503-6510.

24. Lee $Y$, et al. (2008) Gene expression analysis of glioblastomas identifies the major molecular basis for the prognostic benefit of younger age. BMC Med Genomics 1:52. from the QuantiBRITE bead data using the FlowJo Data Analysis software calibration tool.

ACKNOWLEDGMENTS. We thank Libuse Jerabek, Rosalind Ravasio, Donna Mahood, Linda Quinn, Rebecca Broome, Amy Erickson, and Cathy Emory for their laboratory assistance and administrative support; Janet Bruno and Jennifer Santos for assisting in tissue acquisition; Kelli Montgomery for tissue staining and scanning; Susan Prohaska for critical discussions and insightful feedback; the Contag laboratory (Stanford University) for bioluminescence imaging reagents; Eric Brown and Hiroshi Morisaki (Genentech) for providing anti-mouse CD47 hybridomas; and Rosey Mushens (International Blood Group Reference Laboratory, United Kingdom) for production and purification of the Bric126 antibody. This work was supported by The Joseph and Laurie Lacob Gynecologic/Ovarian Cancer Fund, The Jim and Carolyn Pride Fund, The Virginia and D. K. Ludwig Fund for Cancer Research, The Weston Havens Foundation, The National Cancer Institute (5P01CA139490), Department of Defense Award W81XWH-07-1-0467, and anonymous donors.

25. Smith JJ, et al. (2010) Experimentally derived metastasis gene expression profile predicts recurrence and death in patients with colon cancer. Gastroenterology 138: 958-968.

26. Chan KS, et al. (2009) Identification, molecular characterization, clinical prognosis, and therapeutic targeting of human bladder tumor-initiating cells. Proc Natl Acad Sci USA 106:14016-14021.

27. Dalerba $P$, et al. (2007) Phenotypic characterization of human colorectal cancer stem cells. Proc Natl Acad Sci USA 104:10158-10163.

28. Sica A, et al. (2008) Macrophage polarization in tumour progression. Semin Cancer Biol 18:349-355.

29. Allavena P, Sica A, Solinas G, Porta C, Mantovani A (2008) The inflammatory microenvironment in tumor progression: The role of tumor-associated macrophages. Crit Rev Oncol Hematol 66:1-9.

30. Gordon S (2003) Alternative activation of macrophages. Nat Rev Immuno/ 3:23-35.

31. Addison CL, et al. (1995) Intratumoral injection of an adenovirus expressing interleukin 2 induces regression and immunity in a murine breast cancer model. Proc Natl Acad Sci USA 92:8522-8526.

32. Han X, et al. (2000) CD47, a ligand for the macrophage fusion receptor, participates in macrophage multinucleation. J Biol Chem 275:37984-37992.

33. Lindberg FP, Gresham HD, Schwarz E, Brown EJ (1993) Molecular cloning of integrinassociated protein: An immunoglobulin family member with multiple membranespanning domains implicated in alpha $v$ beta 3-dependent ligand binding. J Cell Biol 123:485-496.

34. Ogden CA, et al. (2001) C1q and mannose binding lectin engagement of cell surface calreticulin and CD91 initiates macropinocytosis and uptake of apoptotic cells. J Exp Med 194:781-795.

35. Hoffmann PR, et al. (2001) Phosphatidylserine (PS) induces PS receptor-mediated macropinocytosis and promotes clearance of apoptotic cells. J Cell Biol 155:649-659.

36. Gardai SJ, et al. (2005) Cell-surface calreticulin initiates clearance of viable or apoptotic cells through trans-activation of LRP on the phagocyte. Cell 123:321-334.

37. Chao M, et al. (2010) Calreticulin is the dominant pro-phagocytic signal on multiple human cancers and is counterbalanced by CD47. Sci Transl Med 2:63ra94.

38. Obeid M, et al. (2007) Calreticulin exposure is required for the immunogenicity of gamma-irradiation and UVC light-induced apoptosis. Cell Death Differ 14:1848-1850.

39. Obeid M, et al. (2007) Calreticulin exposure dictates the immunogenicity of cancer cell death. Nat Med 13:54-61.

40. Clynes RA, Towers TL, Presta LG, Ravetch JV (2000) Inhibitory Fc receptors modulate in vivo cytotoxicity against tumor targets. Nat Med 6:443-446.

41. Jemal A, et al. (2008) Cancer statistics, 2008. CA Cancer J Clin 58:71-96.

42. Wyckoff JB, et al. (2007) Direct visualization of macrophage-assisted tumor cell intravasation in mammary tumors. Cancer Res 67:2649-2656.

43. Wang XQ, Frazier WA (1998) The thrombospondin receptor CD47 (IAP) modulates and associates with alpha2 beta1 integrin in vascular smooth muscle cells. Mol Biol Cell 9:865-874.

44. Brown E, Hooper L, Ho T, Gresham H (1990) Integrin-associated protein: A 50-kD plasma membrane antigen physically and functionally associated with integrins. J Cell Biol 111:2785-2794.

45. Condeelis J, Pollard JW (2006) Macrophages: Obligate partners for tumor cell migration, invasion, and metastasis. Cell 124:263-266.

46. Pollard JW (2004) Tumour-educated macrophages promote tumour progression and metastasis. Nat Rev Cancer 4:71-78.

47. Hothorn T, Lausen B (2003) On the exact distribution of maximally selected rank statistics. Comput Stat Data Anal 43:121-137.

48. Dai $\mathrm{M}$, et al. (2005) Evolving gene/transcript definitions significantly alter the interpretation of GeneChip data. Nucleic Acids Res 33:e175. 